\title{
Timing of chronic obstructive pulmonary disease diagnosis in lung cancer prognosis: a clinical and genomic-based study
}

\author{
Jie Dai ${ }^{1,2 \#}$, Yanqi He ${ }^{1,3 \#}$, Kunlatida Maneenil ${ }^{4,5}$, Han Liu ${ }^{1,6}$, Ming Liu ${ }^{1,2}$, Qian Guo ${ }^{1,7}$, Amy C. Bennett ${ }^{1}$, \\ Shawn M. Stoddard ${ }^{1}$, Jason A. Wampfler ${ }^{8}$, Gening Jiang ${ }^{2}$, Ping Yang ${ }^{1}$ \\ ${ }^{1}$ Division of Epidemiology, Department of Health Sciences Research, Mayo Clinic, Rochester, MN, USA; ${ }^{2}$ Department of Thoracic Surgery, \\ Shanghai Pulmonary Hospital, Tongji University School of Medicine, Shanghai, China; ${ }^{3}$ Department of Respiratory and Critical Care Medicine, \\ West China Hospital, Sichuan University, Chengdu, China; ${ }^{4}$ Division of Medical Oncology, Mayo Clinic, Rochester, MN, USA; ${ }^{5}$ Oncology Unit, \\ Department of Medicine, Rajavithi Hospital, College of Medicine, Rangsit University, Bangkok, Thailand; ${ }^{6}$ Department of Respiratory Medicine, \\ the First Hospital of Jilin University, Changchun, China; ${ }^{7}$ Department of Medical Oncology, The Second Affiliated Hospital, Dalian Medical \\ University, Dalian, China; ${ }^{8}$ Division of Biomedical Statistics and Informatics, Mayo Clinic, Rochester, MN, USA \\ Contributions: (I) Conception and design: J Dai, Y He, G Jiang, P Yang; (II) Administrative support: P Yang, G Jiang; (III) Provision of study materials \\ or patients: H Liu, Q Guo, AC Bennett, SM Stoddard; (IV) Collection and assembly of data: J Dai, Y He, K Maneenil, H Liu, AC Bennett, SM \\ Stoddard; (V) Data analysis and interpretation: J Dai, M Liu, Q Guo, JA Wampfler; (VI) Manuscript writing: All authors; (VII) Final approval of \\ manuscript: All authors. \\ "These authors contributed equally to this work. \\ Correspondence to: Ping Yang, MD, PhD. Department of Health Sciences Research, Mayo Clinic, 200 First St SW, Rochester, MN 55905, USA. \\ Email: yang.ping@mayo.edu.
}

Background: A two-phase study (clinical and genomic-based) was conducted to evaluate the effect of timing of chronic obstructive pulmonary disease (COPD) diagnosis on lung cancer outcomes.

Methods: The prognostic influence of COPD was investigated in a clinical cohort of 1,986 patients who received surgery for stage I lung cancer; 823 (41.4\%) of them also had COPD, including $549(27.6 \%)$ incidental COPD (diagnosed within 6-months of lung cancer diagnosis) and 274 (13.8\%) prior COPD (>6 months before lung cancer diagnosis). The genomic variations were analyzed from another cohort of 1,549 patients for association with 384 lung cancer-related single nucleotide polymorphisms (SNPs).

Results: Older age ( $\geq 70$ years), smokers, and respiratory symptoms were independent predictors of incidental COPD in lung cancer (all $\mathrm{P}<0.05$ ). Similar to prior COPD, incidental COPD increased postoperative complications and worsened quality-of-life related to dyspnea (both $\mathrm{P}<0.05$ ). Multivariate Cox regression analysis showed lung cancer survival decreased significantly in incidental COPD (HR, 1.30; 95\% CI, 1.02-1.66), but not in prior COPD (HR, 1.15; 95\% CI, 0.87-1.52). Among prior COPD, median survival showed a trend for being better in those with fewer exacerbations (0-1 vs. $\geq 2$ exacerbation/year; 6.1 vs. 4.1 years; $\mathrm{P}=0.10)$. The SNP-based analysis identified ADCY2:rs52827085 was significantly associated with risk of incidental COPD (OR, 1.76; 95\% CI, 1.30-2.38) and NRXN1:rs1356888 associated with prior COPD complicated with lung cancer (OR, 1.73; 95\% CI, 1.29-2.33).

Conclusions: Different long-term survival and genomic variants were observed between lung cancer patients with incidental and with prior COPD, suggesting timing of COPD diagnosis should be considered in lung cancer clinical management and mechanistic research.

Keywords: Chronic obstructive pulmonary disease (COPD); lung cancer; diagnosis; survival; single nucleotide polymorphism (SNP)

Submitted Sep 05, 2020. Accepted for publication Jan 28, 2021.

doi: $10.21037 /$ tlcr-20-1017

View this article at: http://dx.doi.org/10.21037/tlcr-20-1017 


\section{Introduction}

Chronic obstructive pulmonary disease (COPD) and lung cancer are two of the most important smoking-related diseases worldwide (1), and they are closely linked in terms of clinical manifestations and genetic susceptibility $(2,3)$. COPD was associated with 4-10 folds increased risk of lung cancer (3-5), and a strong association was observed for proximal diagnosis of COPD (i.e., within 6 months of lung cancer diagnosis) (6-8). Meanwhile, several genomewide association (GWA) studies and candidate gene studies have identified a variety of putative single nucleotide polymorphisms (SNPs) involved in the development of both diseases $(9,10)$. SNPs may lead to an amino acid change that causes variation in gene regulation and expression, thus modifying lung cancer risk and/or prognosis.

One recent meta-analysis showed a negative impact of COPD on lung cancer survival (11); however, prognostic factors stemming from COPD, i.e., anti-COPD treatment and COPD acute exacerbation, have not been studied in depth. Although $40-66 \%$ of patients with early-stage non-small cell lung cancer (NSCLC) have coexisting COPD (12-14), most of them are unaware of this comorbid condition; COPD is incidentally diagnosed during preoperative lung cancer workup. Compared to previously-recognized COPD, the impact of incidental COPD in lung cancer is poorly understood. Therefore, the primary aims of this study was to investigate the impact of timing of COPD diagnosis on the prognosis of stage I NSCLC, and the secondary aim was to explore the genomic SNP variations between lung cancer patients with incidental and with prior COPD that may contribute to differing mechanisms leading to both diseases. We present the following article in accordance with the STROBE reporting checklist (available at http://dx.doi.org/10.21037/tlcr-20-1017).

\section{Methods}

\section{Patients and data collection}

The study was conducted in accordance with the Declaration of Helsinki (as revised in 2013). The study was approved by the Mayo Clinic Institutional Review Board (IRB\# 225-99) and informed consent was taken from all the patients. Detailed procedures of patient enrollment, data collection, and follow-up have been reported in previous publications $(15,16)$. In the current study, retrospective analysis was conducted for lung cancer and COPD in two stages (Figure S1). Firstly, we performed a clinical analysis of 1,986 patients with stage I pathologically-confirmed NSCLC who underwent complete resection from January 2000 through December 2014 (the clinical cohort). In parallel, to evaluate different roles in genetic susceptibility in lung cancer patients with incidental COPD and with prior COPD, 1,549 patients with data on timing of COPD diagnosis enrolled in the Mayo Clinic Epidemiology and Genetic of Lung Cancer constituted the SNP cohort, of which 432 (27.9\%) patients overlapped with the clinical cohort.

Pulmonary function test was routinely performed in the pre-operative evaluation. COPD was diagnosed when the ratio of post-bronchodilator forced expiratory volume in 1 second (FEV1) and forced vital capacity (FVC) was less than 0.7 (17). According to the results by Powell et al. (6), COPD diagnosis made within 6 months at lung cancer diagnosis was associated with the highest odds ratio (OR) of lung cancer risk. Therefore, in this study, 'incidental COPD' was defined if the diagnosis was made within 6-months at lung cancer diagnosis, while patients with a history of COPD recorded 6 months or longer preceding lung cancer diagnosis were defined as 'prior COPD'. Postoperative complications were defined as those occurred during hospitalization or within 30 days after operation. Quality of life (QOL) was evaluated by the Lung Cancer Symptom Scale (LCSS) within two years after surgery, and each item was assessed as scales varying from 0 (worst) to 10 (best) (18).

\section{SNP selection and quality control}

A total of 384 SNPs were selected from 332 genes following a review of the lung cancer literature and based on our previous unpublished GWA studies (Table S1) $(9,10)$. Genomic DNA was extracted from peripheral blood using the QIAmp DNA extraction kit (Qiagen) and genotyped in the Mayo Clinic Genomics Shared Resource using a custom-designed Illumina GoldenGate panel.

Quality control was implemented in multiple steps as described previously (10). In brief, SNPs were excluded from subsequent analysis if they had Hardy-Weinberg equilibrium (HWE) $\mathrm{P}$ values less than $10^{-4}$, or minor allele frequencies (MAF) less than 0.01 , or call rates less than $95 \%$ in the SNP cohort. As such, 1 SNP failed genotyping, 10 SNPs deviated from HWE, 4 SNPs had MAF less than $1 \%$, and 16 SNPs had missing call rates more than $5 \%$, resulting 
in 353 SNPs being included in the further analyses.

\section{Statistical analysis}

Data was compared across groups using the chi-square test for categorical variables, and the unpaired $t$-test for continuous variables. Risk factors for an incidental COPD in newly-diagnosed lung cancer were evaluated by the multivariate logistic regression via a backward selection process retaining variables with $\mathrm{P}$ values less than 0.05 . The difference in postoperative QOL between groups was assessed using the $t$-test and a clinically important difference was defined as a greater than 1 point $(18,19)$. Survival curves were generated by the Kaplan-Meier method and differences were assessed by the log-rank test. Variables in univariate analyses were all input in multivariate Cox Proportional Hazard model.

For each SNP that passed quality control thresholds, genotype and allele frequencies in COPD groups and non-COPD group were compared separately using the chi-square test, and corresponding ORs were calculated by univariate logistic regression. To correct for multiple comparisons, q values [a false discovery rate (FDR)-adjusted $\mathrm{P}$ value] were computed for each SNP, and an FDR of $10 \%$ was accepted. All statistical analyses were performed by SAS, v9.3 (SAS Institute).

\section{Results}

\section{Patient characteristics}

In the clinical cohort (Table 1), the mean age was $68.4 \pm$ 9.9 years and $1,048(52.8 \%)$ patients were female. The diagnosis of COPD was identified in 823 (41.4\%) patients, including 549 (27.6\%) patients with incidental and 274 $(13.8 \%)$ with prior COPD. The proportion of incidental COPD in total COPD patients was stable at around $68.4 \%$ during the past 15 years (Figure S2).

\section{Risk factors for incidental COPD in lung cancer}

To characterize lung cancer patients with incidental COPD, univariate logistic regression analysis showed 7 significant predictive factors (Table 2). A subsequent multivariate logistic regression model revealed that $\geq 70$ years of age, male, smokers, presenting dyspnea, cough, and hemoptysis were independent predictors of incidental COPD in stage I NSCLC (all $\mathrm{P}<0.05)$.

\section{Subgroup analyses on COPD status}

Patients with incidental COPD were more likely to receive lobectomy as compared to those with prior COPD (64.6\% vs. $50.7 \%, \mathrm{P}<0.01)$. The rate of postoperative complications was significantly higher in COPD groups than that in nonCOPD group $(27.7 \%$ vs. $16.5 \%, \mathrm{P}<0.01)$, with a similar prevalence between the two COPD statuses (incidental vs. prior: $28.1 \%$ vs. $27.0 \%, \mathrm{P}=0.75)$. Same pattern of association was noted when operative procedure was restricted to lobectomy (Table S2).

Although health-related QOL were not significantly different in overall QOL score between non-COPD, incidental COPD, and prior COPD, specific symptom subscales, i.e., dyspnea symptoms were worse in patients with incidental and prior COPD (both $\mathrm{P}<0.05$ and difference $\geq 1$ ). The associations remained significant when restricted to older (age $\geq 70$ years) patients and smokers (Figure 1).

\section{Impact of COPD on survival}

Kaplan-Meier curves show that patients with COPD have worse overall survival than those without $(\mathrm{P}<0.01$, Figure $2 A)$. In the multivariate Cox proportional hazard model adjusting for pulmonary function and tumor characteristics (Table 3), incidental COPD was significantly associated with decreased overall survival [hazard ratio (HR), 1.30; 95\% confidence interval (CI), 1.02-1.66], while prior COPD was not (HR, 1.15 ; $95 \%$ CI, 0.87-1.52).

To explore the prognostic factors arising from COPD, survival analyses were limited in prior COPD patients with treatment information in their medical records $(n=235)$. Based on the Global Initiative for Chronic Obstructive Lung Disease (GOLD) (17), 27 (11.5\%) patients had mild (GOLD I), 157 (66.8\%) moderate (GOLD II), and 51 $(21.7 \%)$ severe airflow limitation (GOLD III and IV). Most patients $(\mathrm{n}=184,78.3 \%)$ had no history of exacerbation; only $20(8.5 \%)$ patients had frequent exacerbations (defined as $\geq 2$ exacerbations per year). In regard to treatment regimen, $30(12.8 \%)$ received inhaled corticosteroid (ICS), 126 (53.6\%) were offered bronchodilators (beta2agonists and/or muscarinic antagonist), and 25 (10.6\%) had a combination of ICS and bronchodilators. Kaplan-Meier curves show that there is a more apparent decrease in survival as severity of airflow limitation increased $(\mathrm{P}<0.01)$; patients with fewer frequencies of exacerbations $(\mathrm{P}=0.10)$ and receiving ICS $(\mathrm{P}=0.22)$ had a slight better survival, but not reaching statistical threshold of significance (Figure 2B,C,D,E,F). 
Table 1 Patient demographics and clinical characteristics $(\mathrm{N}=1,986)$

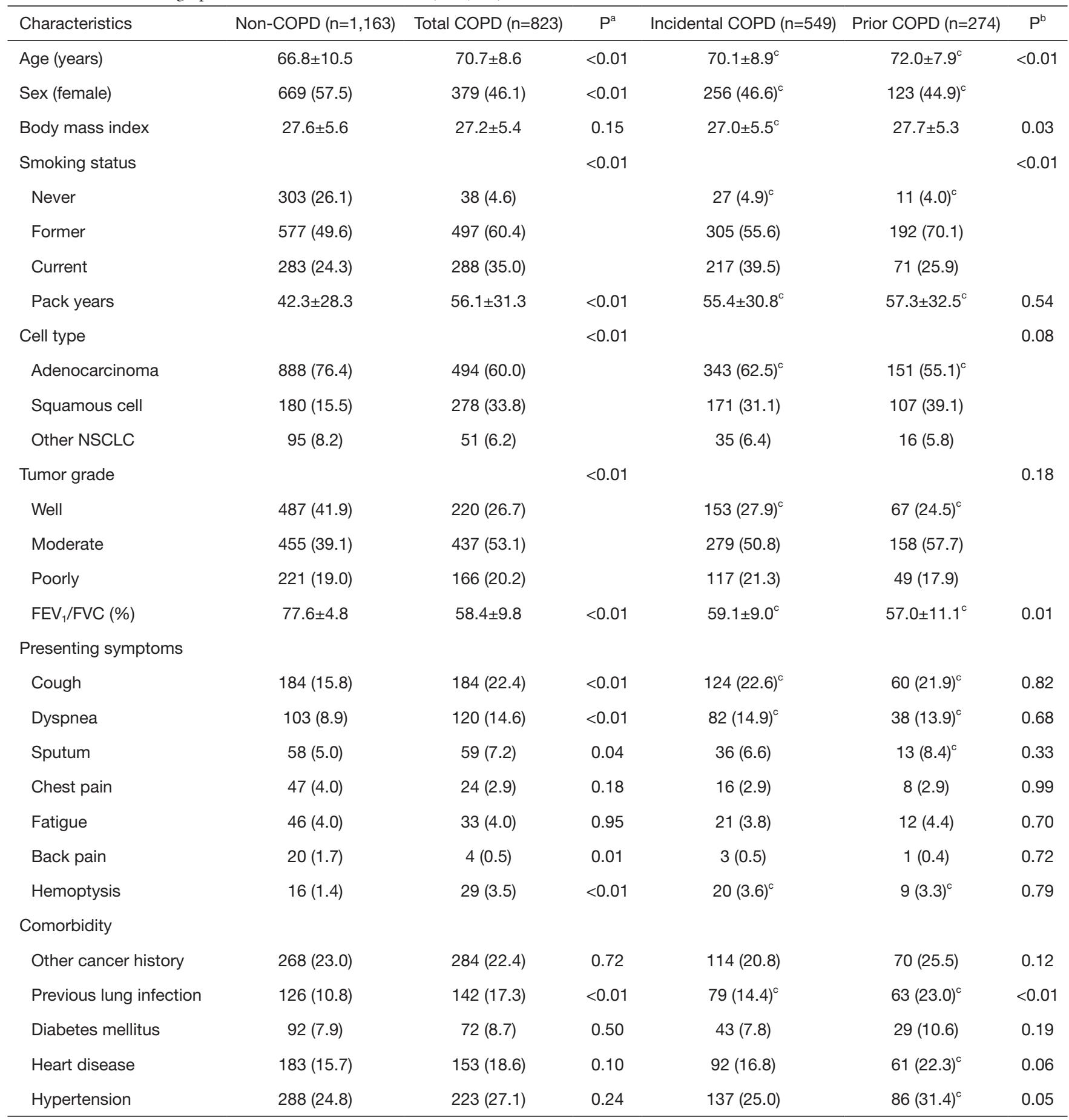

${ }^{a}$, compared with non-COPD; ${ }^{b}$, comparison between incidental and prior COPD; ${ }^{c}$, in comparison to non-COPD with statistically significance. Values are mean \pm SD or $\mathrm{n}(\%)$. NSCLC, non-small cell lung cancer; COPD, chronic obstructive pulmonary disease. 
Table 2 Risk factors for incidentally-diagnosed COPD in lung cancer patients

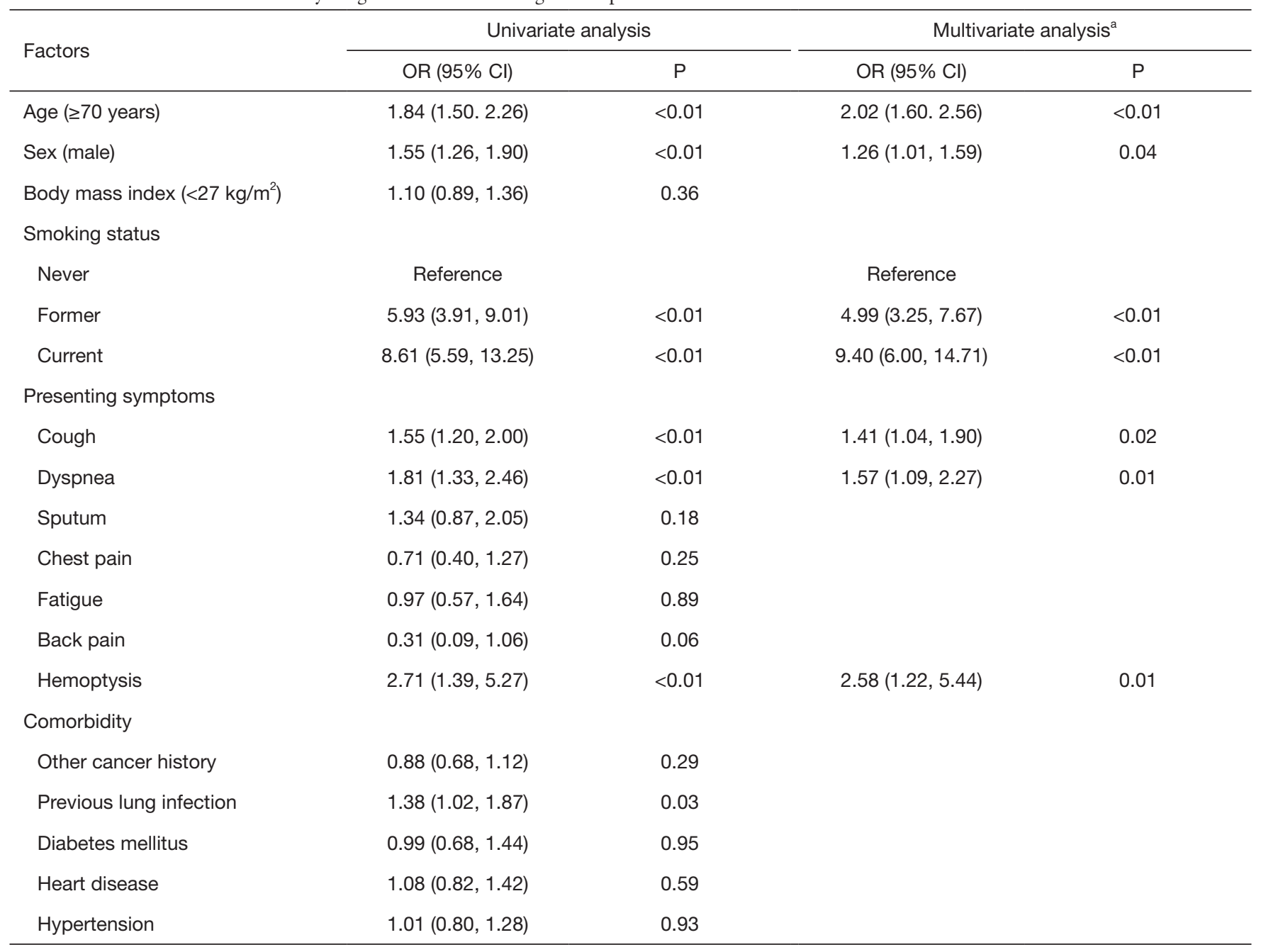

${ }^{a}$, multivariate analysis using backward selection to retain variables with $\mathrm{P}$ values less than 0.05 . OR, odds ratio; $\mathrm{Cl}$, confidence interval; COPD, chronic obstructive pulmonary disease.

\section{Difference in genomic SNP distribution}

In the SNP cohort $(n=1,549)$ patient demographics followed a similar pattern as those in the clinical cohort where patients with COPD were more likely to be male and smokers (Table S3). The genomic analysis identified 18 SNPs in incidental COPD and 15 in prior COPD, with $\mathrm{P}<0.05$, when compared to non-COPD; however, only SNP-rs52827085 in incidental COPD and SNP-rs1356888 in prior COPD passed the FDR $<10 \%$ threshold. The G allele of the SNP-rs52827085 was more frequently observed in patients with incidental COPD $(\mathrm{P}=2.51 \mathrm{E}-04, \mathrm{q}=0.08)$, and the $\mathrm{GG}+\mathrm{GC}$ genotypes were more common among incidental COPD patients $(\mathrm{P}=2.04 \mathrm{E}-04, \mathrm{q}=0.07$, Table 4).
Additionally, genotype distribution (TT+TC versus CC) for SNP-rs1356888 between prior COPD and non-COPD was significantly different $(\mathrm{P}=2.75 \mathrm{E}-04, \mathrm{q}=0.05$, Table 4).

\section{Discussion}

Our investigation revealed a consistent high prevalence of incidental COPD ( $<6$ months of lung cancer diagnosis) among patients with stage I NSCLC during the past 15 years. Timing of COPD diagnosis was associated with lung cancer prognosis where only incidental COPD exerted a significantly negative impact. Two genomic variants (SNPs rs52827085 and rs1356888) were identified that might account for the disparate disease mechanisms of two distinct 
A

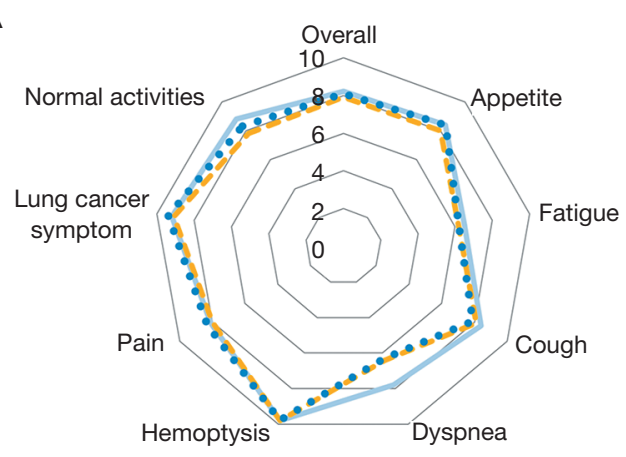

B

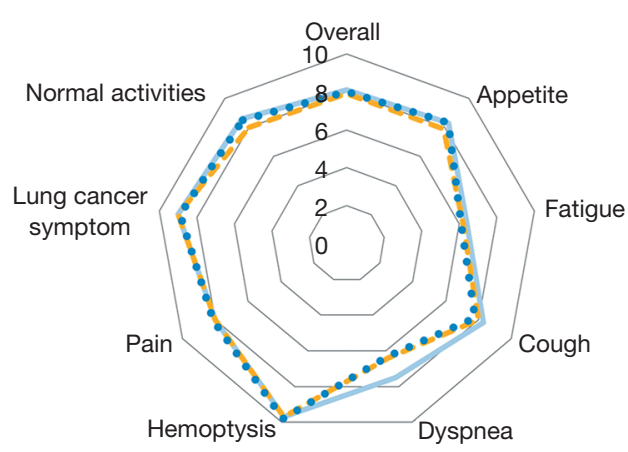

C

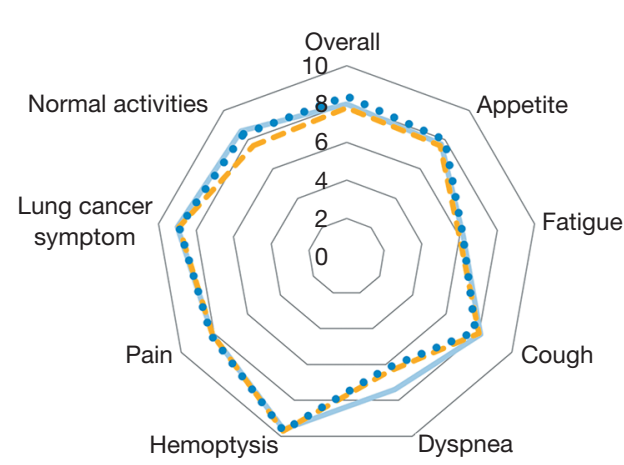

-non-COPD

- -incidental COPD

- prior COPD

\begin{tabular}{|c|c|c|c|}
\hline & \multicolumn{2}{|c|}{ non-COPD incidental COPD } & prior COPD \\
\hline Appetite & $8.4 \pm 1.7$ & $8.0 \pm 1.8$ & $8.4 \pm 1.6$ \\
\hline Fatigue & $6.5 \pm 2.3$ & $6.2 \pm 2.3$ & $6.2 \pm 2.5$ \\
\hline Cough & $8.4 \pm 1.8$ & $8.1 \pm 1.9$ & $7.9 \pm 2.2$ \\
\hline Dyspnea & $7.8 \pm 2.3$ & $6.4 \pm 2.4^{\mathrm{a}}$ & $6.3 \pm 2.5^{\mathrm{a}}$ \\
\hline Hemoptysis & $9.8 \pm 0.6$ & $9.8 \pm 0.4$ & $9.8 \pm 0.2$ \\
\hline Pam & $8.1 \pm 2.2$ & $8.0 \pm 2.3$ & $8.3 \pm 2.2$ \\
\hline Lung cancer symptom & $9.2 \pm 1.5$ & $9.1 \pm 1.4$ & $9.4 \pm 1.9$ \\
\hline Normal activities & $8.8 \pm 1.7$ & $7.8 \pm 2.2$ & $8.3 \pm 2.2$ \\
\hline Overall & $8.2 \pm 1.7$ & $7.9 \pm 1.9$ & $8.1 \pm 1.9$ \\
\hline
\end{tabular}

- non-COPD

- -incidental COPD

-. prior COPD

\begin{tabular}{lccc}
\hline & non-COPD & incidental COPD & prior COPD \\
\hline Appetite & $7.7 \pm 1.8$ & $7.6 \pm 1.7$ & $8.0 \pm 2.0$ \\
Fatigue & $6.2 \pm 2.2$ & $6.0 \pm 2.4$ & $6.2 \pm 2.5$ \\
Cough & $8.1 \pm 1.9$ & $8.0 \pm 1.9$ & $7.8 \pm 2.1$ \\
Dyspnea & $7.4 \pm 2.4$ & $6.4 \pm 2.3^{\mathrm{a}}$ & $6.2 \pm 2.5^{\mathrm{a}}$ \\
Hemoptysis & $9.6 \pm 0.8$ & $9.7 \pm 0.4$ & $9.7 \pm 0.3$ \\
Pam & $8.1 \pm 2.1$ & $8.1 \pm 2.3$ & $8.2 \pm 1.9$ \\
Lung cancer symptom & $9.0 \pm 1.3$ & $8.9 \pm 1.5$ & $8.9 \pm 1.5$ \\
Normal activities & $8.6 \pm 1.6$ & $7.6 \pm 2.4$ & $8.4 \pm 2.3$ \\
Overall & $8.0 \pm 1.8$ & $7.8 \pm 1.7$ & $8.4 \pm 2.0$ \\
\hline
\end{tabular}

-non-COPD

- -incidental COPD

•. prior COPD

\begin{tabular}{|c|c|c|c|}
\hline & \multicolumn{2}{|c|}{ non-COPD incidental COPD } & prior COPD \\
\hline Appetite & $8.3 \pm 1.8$ & $7.9 \pm 1.7$ & $8.3 \pm 1.8$ \\
\hline Fatigue & $6.4 \pm 2.3$ & $6.2 \pm 2.4$ & $6.1 \pm 2.5$ \\
\hline Cough & $8.3 \pm 1.9$ & $8.0 \pm 1.9$ & $7.8 \pm 2.2$ \\
\hline Dyspnea & $7.5 \pm 2.4$ & $6.4 \pm 2.3^{a}$ & $6.3 \pm 2.5^{\mathrm{a}}$ \\
\hline Hemoptysis & $9.7 \pm 0.6$ & $9.7 \pm 0.4$ & $9.8 \pm 0.3$ \\
\hline Pain & $8.0 \pm 2.1$ & $8.0 \pm 2.3$ & $8.1 \pm 2.1$ \\
\hline Lung cancer symptom & $9.0 \pm 1.7$ & $9.0 \pm 1.4$ & $8.9 \pm 1.7$ \\
\hline Normal activities & $8.6 \pm 1.7$ & $8.0 \pm 2.1$ & $8.5 \pm 2.0$ \\
\hline Overall & $8.1 \pm 1.8$ & $7.9 \pm 1.9$ & $8.0 \pm 1.9$ \\
\hline
\end{tabular}

Figure 1 Comparison of postoperative quality of life between non-COPD, incidental COPD and prior COPD. (A) Total patients; (B) patients with age $\geq 70$ years; (C) smokers. Outer circle representing a higher score and better quality of life; ${ }^{\text {a }}$, comparison with non-COPD: $\mathrm{P}<0.05$ and difference $\geq 1$ point.

COPD complicated with lung cancer.

Case-control studies have demonstrated that the association between COPD and lung cancer risk was more significant when a diagnosis of COPD was chronologically close to lung cancer diagnosis $(6,7)$. However, the effect of timing difference on prognosis remains unclear. Our results showed that incidental COPD negatively impacted survival while prior COPD did not. We hypothesized that uncontrolled inflammation and tendency to continue smoking among incidental COPD patients might lead to a decreased survival. Zhang and colleagues found that lung cancer patients with incidental COPD were often undertreated, leaving them at higher risks of having persistent uncontrolled inflammation (20). Our analysis revealed that 
A

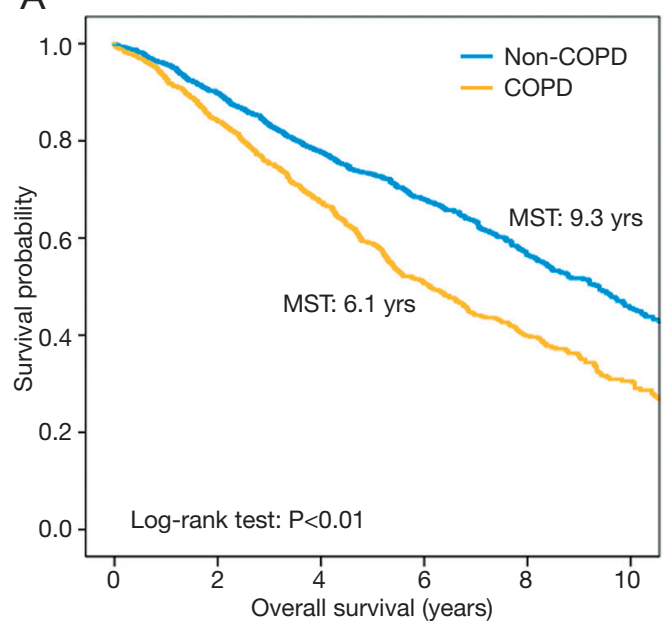

C

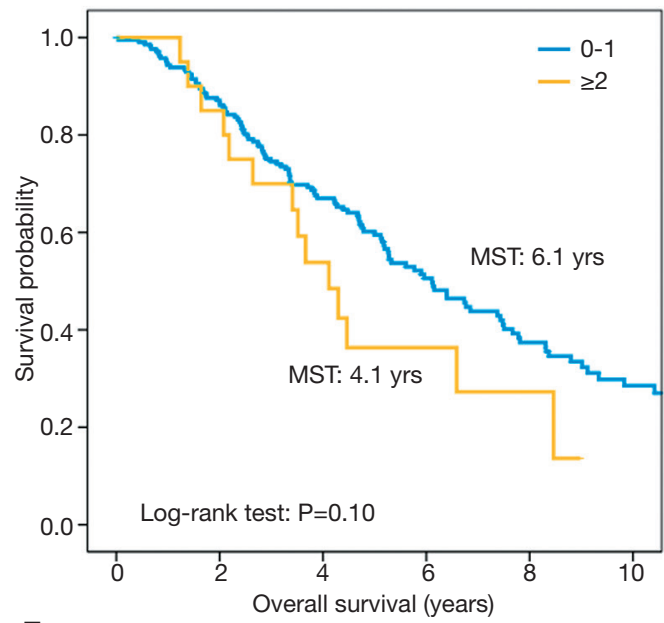

$E$

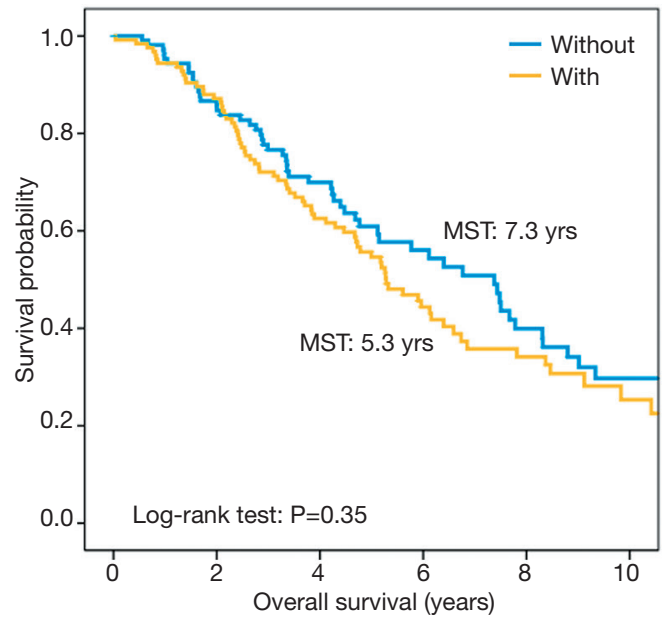

B

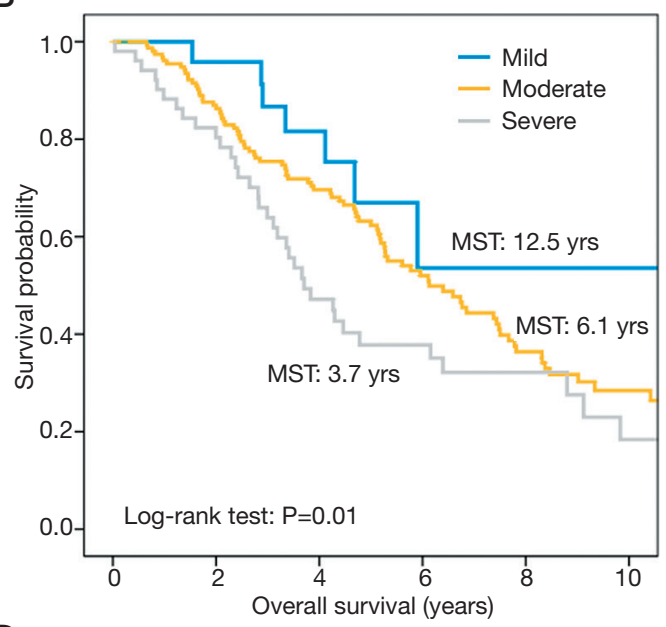

$\mathrm{D}$

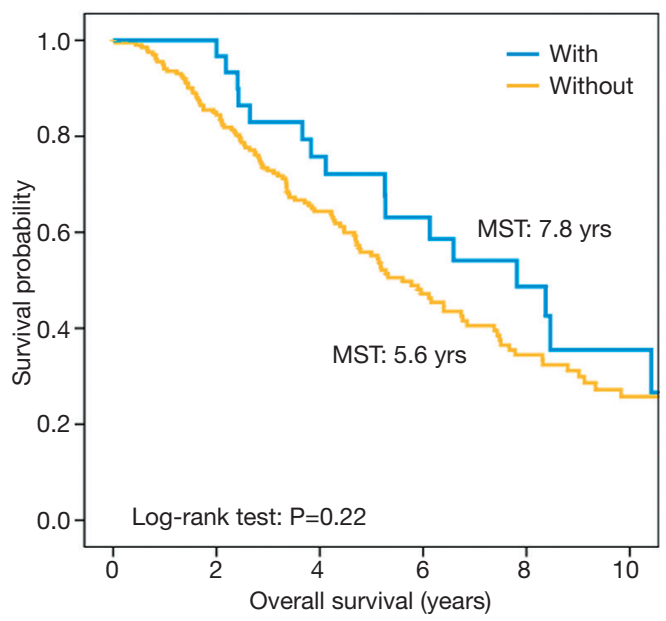

$\mathrm{F}$

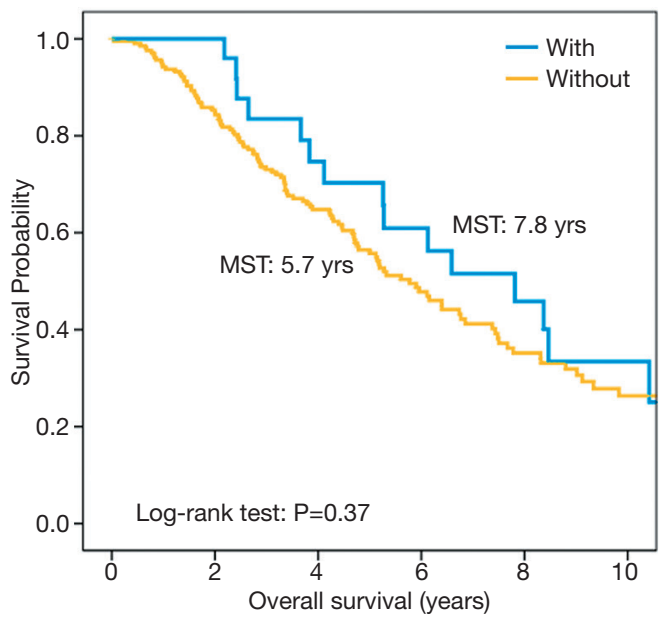

Figure 2 Kaplan-Meier curves for overall survival in total patients (A) and patients with prior COPD (B,C,D,E,F). (A) COPD status; (B) severity of airflow limitation; (C) history of exacerbations per year; (D) inhaled corticosteroid; (E) bronchodilators; (F) both inhaled corticosteroid and bronchodilators. COPD, chronic obstructive pulmonary disease; MST, median survival time. 
Table 3 Cox proportional hazard model for overall survival

\begin{tabular}{|c|c|c|c|c|}
\hline Variables & \multicolumn{2}{|c|}{ Univariate analysis } & \multicolumn{2}{|c|}{ Multivariate analysis $^{a}$} \\
\hline Age & $1.04(1.04,1.05)$ & $<0.01$ & $1.05(1.04,1.06)$ & $<0.01$ \\
\hline Sex (female) & $0.63(0.55,0.71)$ & $<0.01$ & $0.71(0.60,0.83)$ & $<0.01$ \\
\hline Body mass index & $0.99(0.98,1.00)$ & 0.07 & $0.98(0.97,1.00)$ & 0.05 \\
\hline Never & Reference & & Reference & \\
\hline Former & $1.92(1.56,2.38)$ & $<0.01$ & $1.35(1.03,1.77)$ & 0.03 \\
\hline Current & $2.23(1.78,2.78)$ & $<0.01$ & $1.85(1.38,2.49)$ & $<0.01$ \\
\hline \multicolumn{5}{|l|}{ Cell type } \\
\hline Other NSCLC & $1.88(1.51,2.35)$ & $<0.01$ & $1.51(1.12,2.03)$ & $<0.01$ \\
\hline \multicolumn{5}{|l|}{ Tumor grade } \\
\hline Well & Reference & & Reference & \\
\hline Moderate & $1.92(1.64,2.24)$ & $<0.01$ & $1.53(1.26,1.86)$ & $<0.01$ \\
\hline Poorly & $2.11(1.76,2.54)$ & $<0.01$ & $1.64(1.28,2.10)$ & $<0.01$ \\
\hline \multicolumn{5}{|l|}{ Surgical types } \\
\hline Lobectomy & Reference & & Reference & \\
\hline Segmentectomy & $1.67(1.43,1.96)$ & $<0.01$ & $1.52(1.19,1.94)$ & $<0.01$ \\
\hline \multicolumn{5}{|l|}{ COPD status } \\
\hline Prior COPD & $1.58(1.34,1.81)$ & $<0.01$ & $1.15(0.87,1.52)$ & 0.30 \\
\hline
\end{tabular}

a , all variables in univariate analyses were included in multivariate Cox model. HR, hazard ratio; Cl, confidence interval; NSCLC, non-small cell lung cancer; COPD, chronic obstructive pulmonary disease.

fewer COPD exacerbations, a surrogate for decreased airway inflammation, and administration of ICS, which could reduce exacerbation risk and improve lung functional status (17), were associated with a slightly better survival. Several previous studies have also demonstrated a protective effect of ICS on the risk of lung cancer (21); however, these findings should be interpreted with caution, given the small sample size. With respect to smoking habit modification, early COPD diagnosis can help motivate smoking cessation (22), the most effective measure to improve future prospects for the patients (1). As shown in our study, current smokers were more often seen in patients with incidental COPD compared to both non-COPD and prior COPD, which could pose an additional risk of death from lung cancer (23).

We further explore possible molecular mechanistic explanations; the difference in genomic variations was noted between lung cancer patients with incidental and prior COPD, where SNP-rs52827085 was associated with risk of incidental COPD. SNP-rs52827085, located on the intron of adenylate cyclase 2 (ADCY2), is a member of the adenylyl 
Table 4 Genotype and allele frequency for significant single nucleotide polymorphisms

\begin{tabular}{|c|c|c|c|c|c|c|c|c|c|}
\hline Variables & Non-COPD & \multicolumn{4}{|c|}{ Incidental COPD } & \multicolumn{4}{|c|}{ Prior COPD } \\
\hline \multicolumn{10}{|c|}{ ADCY2: rs52827085 } \\
\hline G vs. C & 525 vs. 1,743 & 129 vs. 277 & $1.55(1.23,1.95)$ & $2.51 \mathrm{E}-04$ & 0.08 & 111 vs. 313 & $1.18(0.93,1.49)$ & 1.77E-01 & 0.96 \\
\hline $\mathrm{GG}+\mathrm{GC}$ vs. $\mathrm{CC}$ & 472 vs. 662 & 113 vs. 90 & $1.76(1.30,2.38)$ & $2.04 \mathrm{E}-04$ & 0.07 & 102 vs. 110 & $1.30(0.96,1.75)$ & 7.94E-02 & 0.97 \\
\hline T vs. C & 406 vs. 1,862 & 79 vs. 327 & $1.11(0.85,1.45)$ & 4.53E-01 & 0.93 & 105 vs. 319 & $1.51(1.18,1.93)$ & $9.41 \mathrm{E}-04$ & 0.20 \\
\hline $\mathrm{TT}+\mathrm{TC}$ vs. CC & 362 vs. 772 & 70 vs. 133 & $1.12(0.82,1.54)$ & 4.73E-01 & 0.97 & 95 vs. 117 & $1.73(1.29,2.33)$ & $2.75 \mathrm{E}-04$ & 0.05 \\
\hline
\end{tabular}

a, compared to non-COPD group. Underlined numbers denote significant association at $\mathrm{q}<0.10$. OR, odds ratio; $\mathrm{Cl}$, confidence interval.

cyclase family. Previous GWA studies have identified that ADCY2 was associated with pulmonary function and the ability to quit smoking $(24,25)$, which were concordant to our clinical findings that smoking status and lung function were significantly different between incidental COPD and non-COPD. Furthermore, mutations of ADCY2 were frequently reported in metastatic colorectal cancer, indicating its promoting effect on cancer progression (26). Therefore, it is possible to speculate that ADCY2 gene polymorphism is characterized in incidental COPD and influences lung cancer outcome.

On the other hand, SNP-rs1356888 was significantly associated with prior COPD. It is located on the intron region of NRXN1 gene at chromosome $2 \mathrm{p} 16.3$. The short isoform of NRXN1 could interact with nicotinic acetylcholine receptors (27), and the NRXN1 polymorphism was reportedly associated with lung cancer risk in non-smokers (28). Our findings of these two genes (ADCY2 and NRXN1) indicate their different roles in the development of lung cancer with incidental COPD and with prior COPD, and further functional and mechanistic investigations were needed.

Health-related QOL assessment revealed that COPD was not associated with overall QOL score but with worse postoperative dyspnea, independent of COPD status. Our results corroborate previous works that did not find any association between the presence of COPD and QOL in lung cancer $(29,30)$, indicating that overall QOL level may be mainly affected by lung cancer per se. Dyspnea was the only symptom scored significantly higher in patients with COPD, which is in line with previous knowledge on an obstructive pattern of ventilatory defect in COPD. Moreover, in the present study, patients with COPD tended to be older and smokers, both of which might predispose them to poorer dyspnea (31). Thus, we further did sensitivity analyses in old and smoking patients, and confirmed a similar pattern of difference.

COPD is considered as an independent risk factor for postoperative complications (3). Our results demonstrated that even patients with incidental COPD had a higher incidence of overall postoperative complications than those without, with significant differences in atrial fibrillation, pneumonia, and prolonged air leak (Table S2), and the associations were not influenced by surgical types. Therefore, it behooves surgeons to pay at least as much attention to incidental COPD as to prior COPD during lung cancer work-up.

Since patients with COPD are at higher risk for lung cancer development, early detection of COPD is important for lung cancer surveillance $(4,5)$. We explored the factors for an incidental diagnosis of COPD; patient smoking history and respiratory symptoms (i.e., cough, dyspnea, and hemoptysis) were the most important risk determinants. Of note, dyspnea and cough are the most frequent symptoms of COPD. Since screening for COPD is not recommended in asymptomatic adults (32), our study characterized lung cancer patients who may have incidental (i.e., underdiagnosed) COPD, which in turn may help better estimate COPD burden and select optimal treatment for lung cancer.

To our knowledge, the current study is the first to comprehensively examine the association between timing of COPD diagnosis and lung cancer prognosis with respect to postoperative complications, long-term QOL, and overall survival, and to assess genomic DNA variations between incidental and prior COPD implicated in lung cancer. A high but underappreciated prevalence of COPD, associated with increased risks of postoperative complications, dyspnea, and mortality in lung cancer, underscores the 
importance of correct diagnosis of COPD to improve lung cancer outcomes. However, several limitations should be acknowledged. First, this study was retrospective in nature, though our patient cohort was prospectively followed; thus, the potential bias could not be completely eliminated. Second, the 'incidental COPD' was defined based on previous case-control study of lung cancer risk (6), since there is no acknowledged definition at present. Third, it is difficult to distinguish between COPD and lung cancer as the cause of death, which limits the ability of the study to assess the effects of COPD on lung cancer disease-free survival. Forth, in order to increase the sample size and statistical power, the SNP analysis was not performed on the cohort of patients in the preliminary clinical analysis (only 432 patients overlapped). Therefore, further investigation from larger-scale cohorts is needed to address these issues.

\section{Clinical practice points}

COPD is often concomitant with primary lung cancer and negatively affects lung cancer survival. However, in clinical practice, majority of patients with lung cancer do not recognize their comorbid COPD, and COPD is commonly identified by lung cancer check-ups. It is unclear whether such incidentally-diagnosed COPD plays a different role in lung cancer prognosis, as compared to previouslyrecognized COPD. Focusing on patients with stage I lung cancer, our study has demonstrated that timing of COPD diagnosis matters with respect to lung cancer overall survival, and only incidental COPD exerted a significantly negative impact. Furthermore, a trend was noted for better lung cancer survival in patients with fewer COPD exacerbations and receiving ICS. Meanwhile, an incidental COPD increases the risk of postoperative complications by $12 \%$ and worsens the QOL related to dyspnea. In addition, we further explored the possible genetic mechanisms and found two significant SNP variations between lung cancer with incidental and with prior COPD, where ADCY2:rs52827085 was significantly associated with risk of incidental COPD while NRXN1:rs1356888 associated with prior COPD complicated with lung cancer. These findings indicated that timing of COPD diagnosis should be considered in lung cancer clinical management and mechanistic research.

\section{Conclusions}

In summary, our study demonstrated that timing of COPD diagnosis mattered in the association between COPD and lung cancer prognosis where only incidental COPD was detrimental to the overall survival of lung cancer. In addition, different genomic variants were observed between lung cancer with incidental and with prior COPD, where ADCY2:rs52827085 was associated with incidental COPD while NRXN1:rs1356888 with prior COPD. Further prospective studies with larger numbers of patients and functional analysis are needed to clarify the association of timing of COPD diagnosis with lung cancer prognosis.

\section{Acknowledgments}

The authors thank Ms. Pamela Neville for her professional editing and technical assistance with the manuscript.

Funding: The work was supported by the National Natural Science Foundation of China (Grant No. 81802260), Shanghai Rising-Star Program (Grant No. 19QA1407400), Shanghai "Chen Guang” project (Grant No. 19CG19), and Shanghai Pulmonary Hospital Fund for Excellent Young Scholars (No. fkyq1908), which support the process of data acquisition and statistical analysis.

\section{Footnote}

Reporting Checklist: The authors have completed the STROBE reporting checklist. Available at http://dx.doi. org/10.21037/tlcr-20-1017

Data Sharing Statement: Available at http://dx.doi. org/10.21037/tlcr-20-1017

Conflicts of Interest: All authors have completed the ICMJE uniform disclosure form (available at http://dx.doi. org/10.21037/tlcr-20-1017). The authors have no conflicts of interest to declare.

Ethical Statement: The authors are accountable for all aspects of the work in ensuring that questions related to the accuracy or integrity of any part of the work are appropriately investigated and resolved. The study was conducted in accordance with the Declaration of Helsinki (as revised in 2013). The study was approved by the Mayo Clinic Institutional Review Board (IRB\# 225-99) and informed consent was taken from all the patients.

Open Access Statement: This is an Open Access article distributed in accordance with the Creative Commons 
Attribution-NonCommercial-NoDerivs 4.0 International License (CC BY-NC-ND 4.0), which permits the noncommercial replication and distribution of the article with the strict proviso that no changes or edits are made and the original work is properly cited (including links to both the formal publication through the relevant DOI and the license). See: https://creativecommons.org/licenses/by-nc-nd/4.0/.

\section{References}

1. Thun MJ, Carter BD, Feskanich D, et al. 50-year trends in smoking-related mortality in the United States. N Engl J Med 2013;368:351-64.

2. Houghton AM. Mechanistic links between COPD and lung cancer. Nat Rev Cancer 2013;13:233-45.

3. Dai J, Yang P, Cox A, et al. Lung cancer and chronic obstructive pulmonary disease: From a clinical perspective. Oncotarget 2017;8:18513-24.

4. Young RP, Hopkins RJ, Christmas T, et al. COPD prevalence is increased in lung cancer, independent of age, sex and smoking history. Eur Respir J 2009;34:380-6.

5. Sekine Y, Katsura H, Koh E, et al. Early detection of COPD is important for lung cancer surveillance. Eur Respir J 2012;39:1230-40.

6. Powell HA, Iyen-Omofoman B, Baldwin DR, et al. Chronic obstructive pulmonary disease and risk of lung cancer: the importance of smoking and timing of diagnosis. J Thorac Oncol 2013;8:6-11.

7. Brenner AV, Wang Z, Kleinerman RA, et al. Previous pulmonary diseases and risk of lung cancer in Gansu Province, China. Int J Epidemiol 2001;30:118-24.

8. Schwartz AG, Cote ML, Wenzlaff AS, et al. Chronic obstructive lung diseases and risk of non-small cell lung cancer in women. J Thorac Oncol 2009;4:291-9.

9. Li Y, Sheu CC, Ye Y, et al. Genetic variants and risk of lung cancer in never smokers: a genome-wide association study. Lancet Oncol 2010;11:321-30.

10. de Andrade M, Li Y, Marks RS, et al. Genetic variants associated with the risk of chronic obstructive pulmonary disease with and without lung cancer. Cancer Prev Res (Phila) 2012;5:365-73.

11. Dai J, Liu M, Jiang G, et al. The Impact of Chronic Obstructive Pulmonary Disease on Lung Cancer Survival: A Meta-analysis. Arch Cancer Res 2016;4:1.

12. Zhai R, Yu X, Shafer A, et al. The impact of coexisting COPD on survival of patients with early-stage non-small cell lung cancer undergoing surgical resection. Chest 2014;145:346-53.
13. Lee SJ, Lee J, Park YS, et al. Impact of chronic obstructive pulmonary disease on the mortality of patients with nonsmall-cell lung cancer. J Thorac Oncol 2014;9:812-7.

14. Bugge A, Lund MB, Brunborg C, et al. Survival After Surgical Resection for Lung Cancer in Patients With Chronic Obstructive Pulmonary Disease. Ann Thorac Surg 2016;101:2125-31.

15. Yang P, Allen MS, Aubry MC, et al. Clinical features of 5,628 primary lung cancer patients: experience at Mayo Clinic from 1997 to 2003. Chest 2005;128:452-62.

16. Sun Z, Aubry MC, Deschamps C, et al. Histologic grade is an independent prognostic factor for survival in nonsmall cell lung cancer: an analysis of 5018 hospital- and 712 population-based cases. J Thorac Cardiovasc Surg 2006;131:1014-20.

17. Vogelmeier CF, Criner GJ, Martinez FJ, et al. Global Strategy for the Diagnosis, Management, and Prevention of Chronic Obstructive Lung Disease 2017 Report. GOLD Executive Summary. Am J Respir Crit Care Med 2017;195:557-82.

18. Yang P, Cheville AL, Wampfler JA, et al. Quality of life and symptom burden among long-term lung cancer survivors. J Thorac Oncol 2012;7:64-70.

19. Dai J, Liu M, Swensen SJ, et al. Regional Emphysema Score Predicting Overall Survival, Quality of Life, and Pulmonary Function Recovery in Early-Stage Lung Cancer Patients. J Thorac Oncol 2017;12:824-32.

20. Zhang J, Zhou JB, Lin XF, et al. Prevalence of undiagnosed and undertreated chronic obstructive pulmonary disease in lung cancer population. Respirology 2013;18:297-302.

21. Raymakers AJ, McCormick N, Marra CA, et al. Do inhaled corticosteroids protect against lung cancer in patients with COPD? A systematic review. Respirology 2017;22:61-70.

22. Danielsen SE, Lochen ML, Medbo A, et al. A new diagnosis of asthma or COPD is linked to smoking cessation - the Tromso study. Int J Chron Obstruct Pulmon Dis 2016;11:1453-8.

23. Parsons A, Daley A, Begh R, et al. Influence of smoking cessation after diagnosis of early stage lung cancer on prognosis: systematic review of observational studies with meta-analysis. BMJ 2010;340:b5569.

24. Hancock DB, Eijgelsheim M, Wilk JB, et al. Meta-analyses of genome-wide association studies identify multiple loci associated with pulmonary function. Nat Genet 2010;42:45-52.

25. Uhl GR, Liu QR, Drgon T, et al. Molecular genetics of successful smoking cessation: convergent genome- 
wide association study results. Arch Gen Psychiatry 2008;65:683-93

26. Fang LT, Lee S, Choi H, et al. Comprehensive genomic analyses of a metastatic colon cancer to the lung by whole exome sequencing and gene expression analysis. Int $\mathrm{J}$ Oncol 2014;44:211-21.

27. Nussbaum J, Xu Q, Payne TJ, et al. Significant association of the neurexin-1 gene (NRXN1) with nicotine dependence in European- and African-American smokers. Hum Mol Genet 2008;17:1569-77.

28. Kim JH, Park K, Yim SH, et al. Genome-wide association study of lung cancer in Korean non-smoking women. J Korean Med Sci 2013;28:840-7.

29. Pompili C, Brunelli A, Refai M, et al. Does chronic obstructive pulmonary disease affect postoperative

Cite this article as: Dai J, He Y, Maneenil K, Liu H, Liu M, Guo Q, Bennett AC, Stoddard SM, Wampfler JA, Jiang G, Yang P. Timing of chronic obstructive pulmonary disease diagnosis in lung cancer prognosis: a clinical and genomic-based study. Transl Lung Cancer Res 2021;10(3):1209-1220. doi: 10.21037/ tlcr-20-1017 quality of life in patients undergoing lobectomy for lung cancer? A case-matched study. Eur J Cardiothorac Surg 2010;37:525-30.

30. Mohan A, Mohan C, Pathak AK, et al. Impact of chronic obstructive pulmonary disease on respiratory status and quality of life in newly diagnosed patients with lung cancer. Respirology 2007;12:240-7.

31. Poghosyan H, Sheldon LK, Leveille SG, et al. Healthrelated quality of life after surgical treatment in patients with non-small cell lung cancer: a systematic review. Lung Cancer 2013;81:11-26.

32. Guirguis-Blake JM, Senger CA, Webber EM, et al. Screening for Chronic Obstructive Pulmonary Disease: Evidence Report and Systematic Review for the US Preventive Services Task Force. JAMA 2016;315:1378-93. 


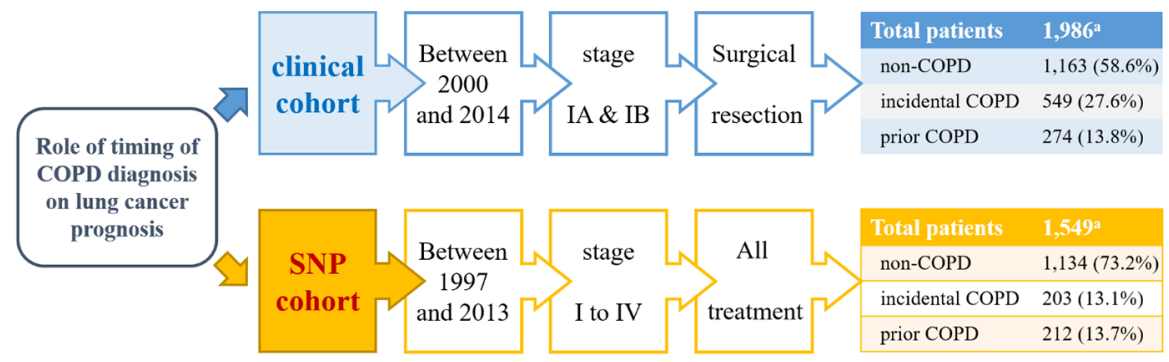

a432 patients overlapped randomly under two independent case retrieving schema.

Figure S1 Patient selection.

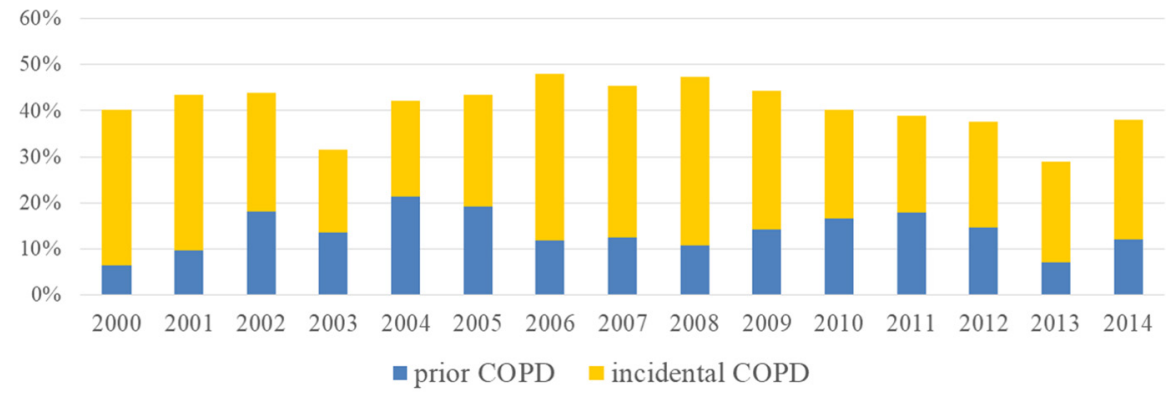

Figure S2 Time trend of rates of incidental and prior COPD in stage I lung cancer. 


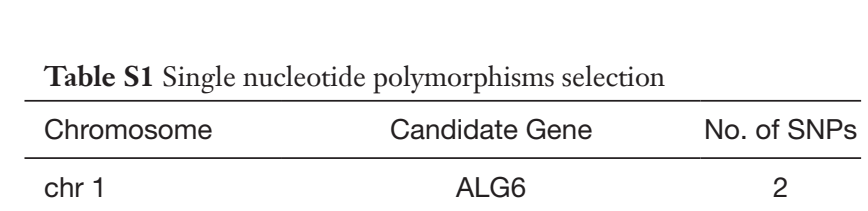

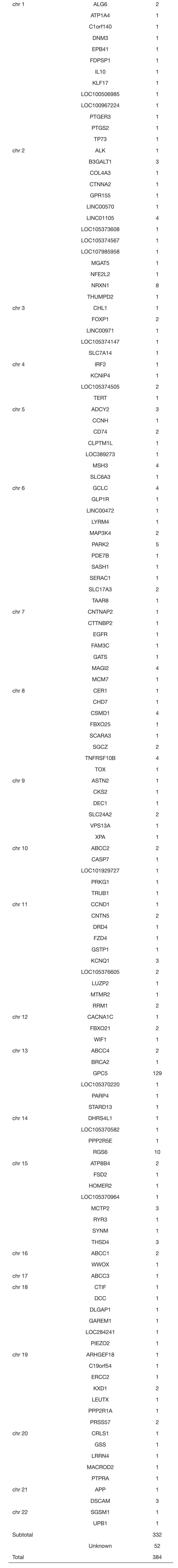


Table S2 Postoperative complications

\begin{tabular}{|c|c|c|c|c|}
\hline \multirow{2}{*}{ Non-COPD, n (\%) } & \multicolumn{2}{|c|}{ Incidental COPD } & \multicolumn{2}{|c|}{ Prior COPD } \\
\hline & n (\%) & $\mathrm{P}^{\mathrm{a}}$ & n (\%) & $\mathrm{P}^{\mathrm{a}}$ \\
\hline
\end{tabular}

\begin{tabular}{lcccccc}
\hline All surgical procedures & & & & & & \\
Any postoperative complications & $192(16.5)$ & $154(28.1)$ & $<0.01$ & $74(27.0)$ & $<0.01$ & 0.75 \\
Atrial fibrillation & $99(8.5)$ & $71(12.9)$ & $<0.01$ & $32(11.7)$ & 0.10 & 0.61 \\
Pneumonia & $21(1.8)$ & $21(3.8)$ & 0.01 & $10(3.6)$ & 0.06 & 0.90 \\
Prolonged air leak & $70(6.0)$ & $71(12.9)$ & $<0.01$ & $36(13.1)$ & $<0.01$ & 0.93 \\
Empyema & $2(0.2)$ & $0(0.0)$ & 0.33 & $1(0.4)$ & 0.53 & 0.16 \\
Chylothorax & $16(1.4)$ & $5(0.9)$ & 0.41 & $3(1.1)$ & 0.71 & 0.80 \\
DVT/PE & $5(0.4)$ & $1(0.2)$ & 0.42 & $1(0.4)$ & 0.88 & 0.62 \\
Perioperative mortality & $6(0.5)$ & $7(1.3)$ & 0.09 & $2(0.7)$ & 0.67 & 0.48 \\
After lobectomy & & & & & \\
Any postoperative complications & $168(19.0)$ & $113(31.8)$ & $<0.01$ & $49(35.5)$ & $<0.01$ & 0.47 \\
Atrial fibrillation & $88(9.9)$ & $53(14.9)$ & 0.01 & $24(17.3)$ & 0.01 & 0.52 \\
Pneumonia & $21(2.4)$ & $17(4.8)$ & 0.03 & $7(5.0)$ & 0.07 & 0.91 \\
Prolonged air leak & $61(6.9)$ & $51(14.4)$ & $<0.01$ & $22(15.8)$ & $<0.01$ & 0.68 \\
Empyema & $2(0.2)$ & $0(0)$ & 0.37 & $1(0.7)$ & 0.32 & 0.11 \\
Chylothorax & $14(1.6)$ & $5(1.4)$ & 0.82 & $3(2.2)$ & 0.62 & 0.55 \\
DVT/PE & $4(0.5)$ & $1(0.3)$ & 0.67 & $0(0)$ & 0.43 & 0.53 \\
Perioperative mortality & $3(0.3)$ & $6(1.7)$ & 0.01 & $0(0)$ & 0.49 & 0.12 \\
\hline
\end{tabular}

${ }^{a}$ compared with non-COPD; ${ }^{b}$ comparison between incidental COPD and prior COPD. Any postoperative complications defined as any complications recorded for each individual patient. DVT/PE: deep vein thrombosis/pulmonary embolism. 
Table S3 Demographics of patients included in the SNP cohort

\begin{tabular}{|c|c|c|c|c|c|}
\hline & Non-COPD $(n=1,134)$ & Incidental COPD $(n=203)$ & Prior COPD (n=212) & Total $(n=1,549)$ & $P$ value \\
\hline Sex & & & & & $<0.01$ \\
\hline Male & $508(44.8)$ & $119(58.6)$ & $108(50.9)$ & 735 (47.4) & \\
\hline Female & $626(55.2)$ & $84(41.4)$ & $104(49.1)$ & $814(52.6)$ & \\
\hline White & $1,033(91.1)$ & $193(95.1)$ & $200(94.3)$ & $1,426(92.1)$ & \\
\hline Non-white & $101(8.9)$ & $10(4.9)$ & $12(5.7)$ & $123(7.9)$ & \\
\hline Smoking status & & & & & $<0.01$ \\
\hline Never & $591(52.1)$ & $30(14.8)$ & $27(12.7)$ & $648(41.8)$ & \\
\hline Histology & & & & & $<0.01$ \\
\hline Adenocarcinoma & $804(70.9)$ & $122(60.1)$ & $144(53.8)$ & $1,040(67.1)$ & \\
\hline Squamous cell & $153(13.5)$ & $59(29.1)$ & $71(33.5)$ & $283(18.3)$ & \\
\hline Other NSCLC & $177(15.6)$ & $22(10.8)$ & $27(12.7)$ & $226(14.6)$ & \\
\hline
\end{tabular}

NSCLC: non-small cell lung cancer. 